\title{
Unsteady Hydromagnetic Heat and Mass Transfer Flow of a Heat Radiating and Chemically Reactive Fluid Past a Flat Porous Plate with Ramped Wall Temperature
}

\author{
R. Nandkeolyar, ${ }^{1}$ M. Das, ${ }^{2}$ and P. Sibanda ${ }^{1}$ \\ ${ }^{1}$ School of Mathematics, Statistics and Computer Science, University of KwaZulu-Natal, Private Bag X01, \\ Scottsville, Pietermaritzburg 3209, South Africa \\ ${ }^{2}$ Department of Mathematics, School of Applied Sciences, KIIT University, Bhubaneswar 751024, India
}

Correspondence should be addressed to P. Sibanda; sibandap@ukzn.ac.za

Received 8 March 2013; Revised 19 July 2013; Accepted 19 July 2013

Academic Editor: Oluwole Daniel Makinde

Copyright (C) 2013 R. Nandkeolyar et al. This is an open access article distributed under the Creative Commons Attribution License, which permits unrestricted use, distribution, and reproduction in any medium, provided the original work is properly cited.

\begin{abstract}
Unsteady hydromagnetic free convective flow of a viscous, incompressible, electrically conducting, and heat radiating fluid past a flat plate with ramped wall temperature and suction/blowing is studied. The governing equations are first subjected to Laplace transformation and then inverted numerically using INVLAP routine of Matlab. The numerical solutions of the fluid properties are presented graphically while the skin friction and heat and mass transfer coefficients are presented in tabular form. The results are verified by a careful comparison with results in the literature for certain parameter values.
\end{abstract}

\section{Introduction}

Magnetohydrodynamic (MHD) free convective flow has attracted many researchers due to its applications in many fluid-engineering problems such as in MHD generators and pumps, plasma studies, nuclear reactors, boundary layer flow control, and geothermal energy extraction. Mention may be made of studies by Gupta [1, 2], Cramer [3], Pop [4], Kuiken [5], Wilks [6], Hossain [7], Aldoss et al. [8], Helmy [9], Kim [10], Takhar et al. [11], and Ahmed et al. [12]. In all these studies, the effects of thermal radiation are not taken into account. However, in industrial applications such as glass production, furnace design, thermonuclear fusion, casting, and levitation and in space technology applications such as cosmical flights, propulsion systems, plasma physics, and space reentry aerodynamics which operate at higher temperature, radiation effect becomes significant. Hossain and Takhar [13] considered the effects of radiation on mixed convection along a vertical plate with uniform temperature. Bakier and Gorla [14] studied the effects of radiation on mixed convection flow over a horizontal surface embedded in a saturated porous medium. Takhar et al. [15] considered the effects of radiation on MHD free convection flow of a gas past a semi-infinite vertical plate. Chamkha [16] studied thermal radiation and buoyancy effects on MHD flow over an accelerating permeable surface with a heat source or sink. Azzam [17] analyzed the effects of radiation on MHD free and forced convection flow past a semi-infinite moving vertical plate with high temperature differences. Israel-Cookey et al. [18] studied the effects of viscous dissipation and radiation on unsteady MHD free convection flow past an infinite heated vertical plate in a porous medium with time dependent suction. Mahmoud [19] studied the thermal radiation effect on unsteady MHD free convection flow of an electrically conducting fluid past an infinite vertical porous plate taking viscous dissipation into account. Bestman and Adjepong [20] investigated unsteady MHD free convection flow of an incompressible optically thick fluid with radiative heat transfer near a moving plate in a rotating medium.

Chamkha et al. [21] investigated radiation effects on free convection flow past a semi-infinite vertical plate in the presence of mass transfer. Thermal radiation effects on nonDarcy free convection flow with lateral mass transfer were 
studied by El-Hakiem and El-Amin [22]. They presented a boundary layer analysis of the influence of thermal radiation and lateral mass flux on non-Darcy natural convection over a vertical flat plate in a fluid saturated porous medium. Prasad et al. [23] investigated the effects of radiation and mass transfer on two-dimensional flow past an impulsively started infinite vertical plate. Makinde and Ogulu [24] considered the heat and mass transfer flows of a variable viscosity fluid past a vertical porous plate permeated by a transverse magnetic field with thermal radiation effects. The effects of thermal radiation and variable fluid viscosity on free convective flow and heat transfer past a porous stretching surface are investigated by Mukhopadhyay and Layek [25]. Pal [26] studied heat and mass transfer in a stagnation point flow towards a stretching surface in the presence of buoyancy force and thermal radiation.

Combined heat and mass transfer flow in the presence of chemically reactive species concentration with or without applied magnetic field has bearing on many transport processes present in nature and also in science and engineering applications. In processes such as drying, evaporation, energy transfer in a cooling tower, and the flow in a desert cooler, heat and mass transfer occur simultaneously. Free convection processes of involving the combined mechanism are also encountered in many natural processes and industrial applications, such as in the curing of plastics, the cleaning and chemical processing of materials, and the manufacturing of pulp and insulated cables. Chamkha [27] studied MHD flow over a uniformly stretched vertical permeable surface subject to a chemical reaction. Afify [28] analyzed the MHD free convective flow and mass transfer over a stretching sheet with a homogeneous chemical reaction of order $n$ where $n$ was taken to be $0,1,2$, or 3 . The influence of a chemical reaction on heat and mass transfer from vertical surfaces in porous media subject to Soret and Dufour effects was studied by Postelnicu [29]. He showed that the thickness of the concentration boundary layer decreases as the Lewis number increases, a phenomenon also evident when a chemical reaction is absent. Kandasamy and Palanimani [30] studied the effects of a chemical reaction on heat and mass transfer on a magnetohydrodynamic boundary layer flow over a wedge with ohmic heating and viscous dissipation in a porous medium. Pal and Mondal [31] studied the effects of Soret Dufour, chemical reaction, and thermal radiation on MHD non-Darcy unsteady mixed convective heat and mass transfer over a stretching sheet.

In all the above investigations, the analytical or numerical solution is obtained assuming that the temperature at the interface was continuous and well defined. However, there exist several problems of physical interest which may require nonuniform or arbitrary wall conditions. Several researchers [32-35] have investigated free convection from a vertical plate with stepdiscontinuities in the surface temperature. Chandran et al. [36] considered unsteady natural convection flow of a viscous incompressible fluid near a vertical plate with ramped wall temperature. Seth et al. [37] studied unsteady natural convection flow of a viscous incompressible electrically conducting fluid past an impulsively moving vertical plate in a porous medium with ramped wall temperature taking into account the effects of thermal radiation. They compared the results of natural convection near a ramped temperature plate with those of natural convection near an isothermal plate. Recently, Seth et al. [38] extended this problem to include the effects of rotation.

Suction or blowing through the wall plays important role in boundary layer formation. An increase in suction velocity may lead to a delay in the formation of a boundary layer and an increase in blowing may lead to a decrease in the skin friction at the plate which, in turn, may lead to a decrease in the rate of heat transfer at the plate. Makinde [39] studied the effects of wall porosity on free convection flow with thermal radiation and mass transfer. Mbeledogu and Ogulu [40] studied the heat and mass transfer rates in rotating flow past a vertical porous flat plate. Afify [41] discussed the MHD free convective heat and mass transfer flow over a stretching sheet in the presence of suction/injection with thermal diffusion and diffusion thermoeffects.

The aim of the present study is to investigate the effects of suction/blowing on unsteady hydromagnetic free convective flow and mass transfer of a viscous, incompressible, electrically conducting, and heat radiating fluid past a flat plate with ramped wall temperature in the presence of chemical reaction. Such a fluid flow finds many engineering applications such as those in MHD devices and in several natural phenomena occurring subject to radiation in the presence of chemically reactive species.

\section{Problem Formulation}

Consider the unsteady free convective heat and mass transfer flow of a viscous, incompressible, electrically conducting, and heat radiating fluid past an impulsively moving vertical porous flat plate. We choose the coordinate system in such a way that the $x$-axis is along the plate in the upward direction, the $y$-axis normal to the plate, and the $z$-axis perpendicular to the $x y$-plane. The fluid is permeated by a uniform transverse magnetic field $B_{0}$ applied parallel to $y$-axis. Initially, at time $t^{\prime} \leq 0$, the fluid and plate are at rest at a uniform temperature $T_{\infty}^{\prime}$ and species concentration $C_{\infty}^{\prime}$. At time $t^{\prime}>0$, the plate begins to move in the $x$-direction with uniform velocity $U_{0}$. Instantaneously, the plate temperature is raised or lowered to $T_{\infty}^{\prime}+\left(T_{w}^{\prime}-T_{\infty}^{\prime}\right) t^{\prime} / t_{0}$ when $t^{\prime}<t_{0}$, and for $t^{\prime}>t_{0}$ the plate is maintained at constant temperature $T_{w}^{\prime}$. A constant species concentration $C_{w}^{\prime}$ is maintained at the plate for $t^{\prime}>0$. Since the plate is of infinite extent in the $x$-and $z$-directions and is electrically nonconducting, all physical quantities, except the pressure, are functions of $y$ and $t^{\prime}$ only.

The fluid is a metallic liquid whose magnetic Reynolds number is small and hence the induced magnetic field produced by the fluid motion is negligible in comparison to the applied one [42] so that the magnetic field $\mathbf{B}=\left(0, B_{0}, 0\right)$. Also, no external electric field is applied so the effect of polarization of magnetic field is negligible [43]; that is, $\mathbf{E}=$ $(0,0,0)$. The fluid flow is induced by the impulsive movement of the plate in the $x$-direction, and there is constant flow of fluid in $y$-direction due to the pores in the plate so that the 
velocity vector is $\mathbf{q}=\left(u,-v_{0}, 0\right)$. With these assumptions, the governing model equations are given by

$$
\begin{aligned}
& \frac{\partial u^{\prime}}{\partial t^{\prime}}-v_{0} \frac{\partial u^{\prime}}{\partial y}= \nu \frac{\partial^{2} u^{\prime}}{\partial y^{2}}-\frac{\sigma B_{0}^{2}}{\rho} u^{\prime} \\
&+g \beta_{T}\left(T^{\prime}-T_{\infty}^{\prime}\right)+g \beta_{C}\left(C^{\prime}-C_{\infty}^{\prime}\right) \\
& \frac{\partial T^{\prime}}{\partial t^{\prime}}-v_{0} \frac{\partial T^{\prime}}{\partial y}=\frac{k}{\rho c_{p}} \frac{\partial^{2} T^{\prime}}{\partial y^{2}}-\frac{1}{\rho c_{p}} \frac{\partial q_{r}^{\prime}}{\partial y^{\prime}} \\
& \frac{\partial C^{\prime}}{\partial t^{\prime}}-v_{0} \frac{\partial C^{\prime}}{\partial y}=D \frac{\partial^{2} C^{\prime}}{\partial y^{\prime 2}}-k_{r}\left(C^{\prime}-C_{\infty}^{\prime}\right)
\end{aligned}
$$

where $u^{\prime}, v_{0}, \rho, g, \beta_{T}, \beta_{C}, T^{\prime}, C^{\prime}, c_{p}, k, q_{r}, v, \sigma, D$, and $k_{r}$ are, respectively, fluid velocity in $x$-direction, suction/injection velocity in $y$ direction, fluid density, acceleration due to gravity, volumetric coefficient of thermal expansion, volumetric coefficient of expansion or contraction, temperature of the fluid near the plate, species concentration, specific heat at constant pressure, thermal conductivity, radiative flux, kinematic coefficient of viscosity, electrical conductivity, chemical molecular diffusivity, and chemical reaction coefficient.

Assuming that there is no slip between the plate and the fluid, the initial and boundary conditions for the fluid flow problem are

$$
\begin{gathered}
u^{\prime}=0, \quad T^{\prime}=T_{\infty}^{\prime}, \quad C^{\prime}=C_{\infty}^{\prime} \quad \text { for } y \geq 0, t^{\prime} \leq 0, \\
u^{\prime}=U_{0} \quad \text { at } y=0 \text { for } t^{\prime}>0, \\
T^{\prime}=T_{\infty}^{\prime}+\frac{\left(T_{w}^{\prime}-T_{\infty}^{\prime}\right) t^{\prime}}{t_{0}} \quad \text { at } y=0 \text { for } 0<t^{\prime} \leq t_{0}, \\
T^{\prime}=T_{w}^{\prime} \quad \text { at } y=0 \text { for } t^{\prime}>t_{0}, \\
C^{\prime}=C_{w}^{\prime} \quad \text { at } y=0 \text { for } t^{\prime}>0, \\
u^{\prime} \longrightarrow 0, \quad T^{\prime} \longrightarrow T_{\infty}^{\prime}, \quad C^{\prime} \longrightarrow C_{\infty}^{\prime} \\
\text { as } y \longrightarrow \infty \text { for } t^{\prime}>0 .
\end{gathered}
$$

For an optically thick fluid, in addition to emission there is also self-absorption, and usually the absorption coefficient is wavelength dependent and large so that we can adopt the Rosseland approximation for radiative flux vector $q_{r}^{\prime}$. The radiative flux vector $q_{r}^{\prime}$ under the Rosseland approximation is

$$
q_{r}^{\prime}=-\frac{4 \sigma^{*}}{3 k^{*}} \frac{\partial T^{\prime 4}}{\partial y}
$$

where $k^{*}$ is the mean absorption coefficient and $\sigma^{*}$ is the Stefan-Boltzmann constant. Assuming a small temperature difference between the fluid temperature $T^{\prime}$ and the free stream temperature $T_{\infty}^{\prime}, T^{\prime 4}$ is expanded in a Taylor series about the free stream temperature $T_{\infty}^{\prime}$. Neglecting second and higher order terms in $\left(T^{\prime}-T_{\infty}^{\prime}\right)$, we obtain

$$
T^{\prime 4} \cong 4 T_{\infty}^{\prime 3} T^{\prime}-3 T_{\infty}^{\prime 4} .
$$

Using (5) and (6), in (2), we obtain

$$
\frac{\partial T^{\prime}}{\partial t^{\prime}}-v_{0} \frac{\partial T^{\prime}}{\partial y}=\frac{k}{\rho c_{p}} \frac{\partial^{2} T^{\prime}}{\partial y^{2}}+\frac{1}{\rho c_{p}} \frac{16 \sigma^{*} T_{\infty}^{\prime 3}}{3 k^{*}} \frac{\partial^{2} T^{\prime}}{\partial y^{2}} .
$$

Introducing the following dimensionless variables

$$
\begin{aligned}
& \eta=\frac{y^{\prime}}{U_{0} t_{0}}, \quad u=\frac{u^{\prime}}{U_{0}}, \quad t=\frac{t^{\prime}}{t_{0}}, \\
& T=\frac{T^{\prime}-T_{\infty}^{\prime}}{T_{w}^{\prime}-T_{\infty}^{\prime}}, \quad C=\frac{C^{\prime}-C_{\infty}^{\prime}}{C_{w}^{\prime}-C_{\infty}^{\prime}},
\end{aligned}
$$

the governing equations (1), (3), and (7) in dimensionless form become

$$
\begin{gathered}
\frac{\partial u}{\partial t}-S \frac{\partial u}{\partial \eta}=\frac{\partial^{2} u}{\partial \eta^{2}}+T+\mathrm{GmC}-M u, \\
\frac{\partial T}{\partial t}-S \frac{\partial T}{\partial \eta}=\frac{(1+N)}{\operatorname{Pr}} \frac{\partial^{2} T}{\partial \eta^{2}}, \\
\frac{\partial C}{\partial t}-S \frac{\partial C}{\partial \eta}=\frac{1}{S_{c}} \frac{\partial^{2} C}{\partial \eta^{2}}-K_{r} C,
\end{gathered}
$$

where $S=v_{0} / U_{0}$ is the suction or blowing parameter, $\mathrm{Gm}=$ $g \beta_{C} \nu\left(C_{w}^{\prime}-C_{\infty}^{\prime}\right) / U_{0}^{3}$ is the mass Grashof number, $M=$ $\sigma B_{0}^{2} \nu / \rho U_{0}^{2}$ is the magnetic parameter, $N=16 \sigma^{*} T_{\infty}^{\prime 3} / 3 k k^{*}$ is the thermal radiation parameter, $\operatorname{Pr}=\rho \nu c_{p} / k$ is the Prandtl number, Sc $=\nu / D$ is the Schmidt number, and $K_{r}=k_{r} \nu^{2} / D U_{0}^{2}$ is the chemical reaction parameter. Here, $S>0$ corresponds to suction, whereas $S<0$ corresponds to blowing. The characteristic time $t_{0}$ and the characteristic velocity $U_{0}$ are defined as

$$
t_{0}=\frac{v}{U_{0}^{2}}, \quad U_{0}=\left[g \beta_{T} v\left(T_{w}^{\prime}-T_{\infty}^{\prime}\right)\right]^{1 / 3} .
$$

The initial and boundary conditions (4a)-(4f) in nondimensional form are

$$
\begin{gathered}
u=0, \quad T=0, \quad C=0 \text { for } \eta \geq 0, t \leq 0, \\
u=1, \quad C=1 \quad \text { at } \eta=0 \text { for } t>0, \\
T=t \quad \text { at } \eta=0 \text { for } 0<t \leq 1, \\
T=1 \text { at } \eta=0 \text { for } t>1, \\
u \longrightarrow 0, \quad T \longrightarrow 0, \quad C \longrightarrow 0 \text { as } \eta \longrightarrow \infty \text { for } t>0 .
\end{gathered}
$$

The system of partial differential equations (9) subject to the initial and boundary conditions (11a)-(11e) represents the model for unsteady hydromagnetic free convective flow of a viscous, incompressible, electrically conducting, and heat radiating fluid past an infinite porous flat plate with ramped wall temperature in the presence of a chemically reactive species. 
TABLE 1: A comparison of $-\delta T /\left.\delta \eta\right|_{\eta=0}$ when $S=0$.

\begin{tabular}{cccc}
\hline$t$ & $N$ & Seth et al. [37] & Present results \\
\hline \multirow{4}{*}{0.2} & 0.5 & 0.3472 & 0.347179 \\
& 1 & 0.3007 & 0.300666 \\
& 5 & 0.1736 & 0.173590 \\
& 10 & 0.1282 & 0.128204 \\
\hline \multirow{4}{*}{0.4} & 0.5 & 0.4910 & 0.490985 \\
& 1 & 0.4252 & 0.425206 \\
& 5 & 0.2455 & 0.245493 \\
& 10 & 0.1813 & 0.181308 \\
\hline \multirow{3}{*}{0.6} & 0.5 & 0.6013 & 0.601332 \\
& 1 & 0.5208 & 0.520769 \\
& 5 & 0.3007 & 0.300666 \\
& 10 & 0.2221 & 0.222057 \\
\hline \multirow{3}{*}{0.8} & 0.5 & 0.6944 & 0.694358 \\
& 1 & 0.6013 & 0.601332 \\
& 5 & 0.3472 & 0.347179 \\
& 10 & 0.2564 & 0.256409 \\
\hline
\end{tabular}

\section{Analytic Solutions}

Using the Laplace transform technique on the system of equations (9), subject to the initial and boundary conditions (11a)-(11e), we obtain

$$
\begin{gathered}
\tilde{u}(\eta, s)=-\left[\left\{\frac{\mathrm{Gm}}{s D_{1}}\right\} e^{\lambda_{1} \eta}+\left\{\frac{\left(1-e^{-s}\right)}{s^{2} D_{2}}\right\} e^{\lambda_{2} \eta}\right] \\
+\left\{\frac{1}{s}+\frac{\left(1-e^{-s}\right)}{s^{2} D_{1}}+\frac{G m}{s D_{2}}\right\} e^{\lambda_{3} \eta}, \\
\widetilde{T}(\eta, s)=\frac{\left(1-e^{-s}\right)}{s^{2}} e^{\lambda_{2} \eta}, \\
\widetilde{C}(\eta, s)=\frac{e^{\lambda_{1} \eta}}{s},
\end{gathered}
$$

where $\widetilde{C}(\eta, s), \widetilde{T}(\eta, s), \widetilde{u}(\eta, s)$ are, respectively, the Laplace transforms of $C(\eta, t), T(\eta, t)$, and $u(\eta, t), s>0$ is the Laplace transform parameter, and

$$
\begin{gathered}
\lambda_{1}=0.5\left\{-S S c-\sqrt{S^{2} S c^{2}+4 S c\left(K_{r}+s\right)}\right\}, \\
\lambda_{2}=0.5\left\{-S\left(\frac{\operatorname{Pr}}{1+N}\right)-\sqrt{\left.S^{2}\left(\frac{\operatorname{Pr}}{1+N}\right)^{2}+4\left(\frac{P r}{1+N}\right) s\right\},}\right. \\
\lambda_{3}=0.5\left\{-S-\sqrt{S^{2}+4(M+s)}\right\}, \\
D_{1}=\lambda_{1}^{2}+S \lambda_{1}-(M+s), \\
D_{2}=\lambda_{2}^{2}+S \lambda_{2}-(M+s) .
\end{gathered}
$$

An exact Laplace transform inversion of (12) can be obtained when there is no suction/blowing, that is, when
$S=0$. However, for a nonzero $S$ the inversion of (12) is not possible. Thus, the inversion of (12)-(14) was obtained numerically using INVLAP routine in Matlab. However, to compare the results which are obtained using INVLAP routine, the exact inversion of (13) and (14) gave the solutions

$$
\begin{gathered}
T(\eta, t)=e^{-b \eta}[P(\eta, t)-H(t-1) P(\eta, t-1)], \\
C(\eta, t)=\frac{1}{2}\left[e^{-\eta(a-\sqrt{d})} \operatorname{erfc}\left(t_{1}\right)+e^{-\eta(a+\sqrt{d})} \operatorname{erfc}\left(t_{2}\right)\right],
\end{gathered}
$$

where erfc is the complementary error function, $H$ is the Heaviside unit step function, and

$$
\begin{aligned}
& P(\eta, t)=\left(\frac{t}{2}+\frac{m \eta}{4 \sqrt{n}}\right) e^{\eta \sqrt{n}} \operatorname{erfc}\left(t_{3}\right) \\
& +\left(\frac{t}{2}-\frac{m \eta}{4 \sqrt{n}}\right) e^{-\eta \sqrt{n}} \operatorname{erfc}\left(t_{4}\right) \text {; } \\
& t_{1}, t_{2}= \pm \sqrt{\frac{d t}{c}}+\frac{\eta}{2} \sqrt{\frac{c}{t}} ; \quad t_{3}, t_{4}= \pm \sqrt{\frac{n t}{m}}+\frac{\eta}{2} \sqrt{\frac{m}{t}} \\
& a=\frac{S S c}{2}, \quad b=\frac{\operatorname{PrS}}{2(1+N)}, \quad c=\mathrm{Sc}, \\
& d=\frac{S^{2} \mathrm{Sc}^{2}}{4}+\mathrm{Sc} K_{r}, \quad m=\frac{\operatorname{Pr}}{1+N}, \\
& n=\frac{\operatorname{Pr}^{2} S^{2}}{4(1+N)^{2}}
\end{aligned}
$$

In the absence of suction/blowing and the thermal radiation effect $(S=0$ and $N=0)$, the solution $T(\eta, t)$ in (16) is in agreement with the solution obtained by Chandran et al. [36].

3.1. Skin Friction, Nusselt Number, and Sherwood Number. The nondimensional quantities of engineering interest are the skin friction $\tau$, which is a measure of shear stress at the plate, the Nusselt number $\mathrm{Nu}$, which measures the rate of heat transfer, and the Sherwood number Sh, which measures the rate of mass transfer at the plate. The Nusselt number $\mathrm{Nu}$ and the Sherwood number Sh have the exact values

$$
\begin{aligned}
\frac{\mathrm{Nu}}{\operatorname{Re}}=- & (1+N)\left(\frac{\partial T}{\partial \eta}\right)_{\eta=0}=-[f(t)-H(t-1) f(t-1)], \\
\frac{\mathrm{Sh}}{\operatorname{Re}} & =-\left(\frac{\partial C}{\partial \eta}\right)_{\eta=0} \\
& =(a+\sqrt{d})+\sqrt{\frac{c}{\pi t}} e^{-d t / c}-\sqrt{d} \operatorname{erfc}\left(\sqrt{\frac{d t}{c}}\right),
\end{aligned}
$$


TABLE 2: Comparison of exact and numerical values of $\mathrm{Nu} / \mathrm{Re}$ and $\mathrm{Sh} / \mathrm{Re}$ for different values of $t$ and $S$ when $K_{r}=0.5, \mathrm{Sc}=1, \mathrm{Pr}=0.71$, and $N=1$.

\begin{tabular}{|c|c|c|c|c|c|}
\hline \multirow{2}{*}{$t$} & \multirow{2}{*}{$S$} & $\mathrm{Nu} / \mathrm{Re}$ & $\mathrm{Nu} / \mathrm{Re}$ & $\mathrm{Sh} / \mathrm{Re}$ & $\mathrm{Sh} / \mathrm{Re}$ \\
\hline & & Exact & Numerical & Exact & Numerical \\
\hline \multirow{3}{*}{0.2} & -1 & 0.53388346 & 0.533883 & 0.94620854 & 0.946209 \\
\hline & 0 & 0.60133195 & 0.601332 & 1.38566159 & 1.385662 \\
\hline & 1 & 0.67588346 & 0.675883 & 1.94620854 & 1.946209 \\
\hline \multirow{3}{*}{0.4} & -1 & 0.71843936 & 0.718439 & 0.64706152 & 0.647062 \\
\hline & 0 & 0.85041184 & 0.850412 & 1.06475703 & 1.064757 \\
\hline & 1 & 1.00243936 & 1.002439 & 1.64706152 & 1.647062 \\
\hline \multirow{3}{*}{0.6} & -1 & 0.84692696 & 0.846927 & 0.53359416 & 0.533594 \\
\hline & 0 & 1.04153758 & 1.041537 & 0.93657181 & 0.936572 \\
\hline & 1 & 1.27292696 & 1.272927 & 1.53359416 & 1.533594 \\
\hline \multirow{3}{*}{0.8} & -1 & 0.94692675 & 0.946927 & 0.47550301 & 0.475503 \\
\hline & 0 & 1.20266405 & 1.202664 & 0.86753072 & 0.867531 \\
\hline & 1 & 1.51492675 & 1.514927 & 1.47550301 & 1.475503 \\
\hline
\end{tabular}

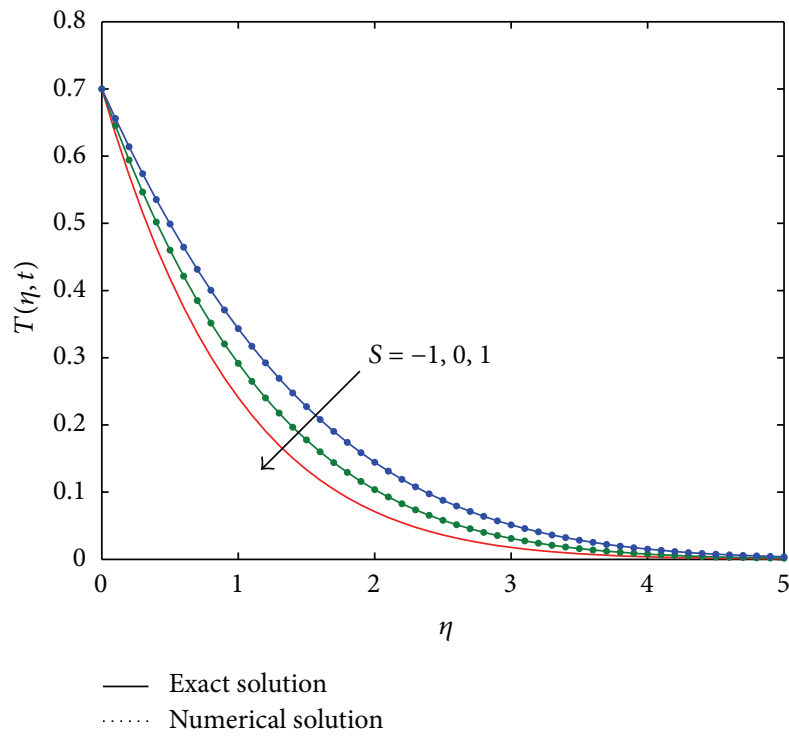

(a)

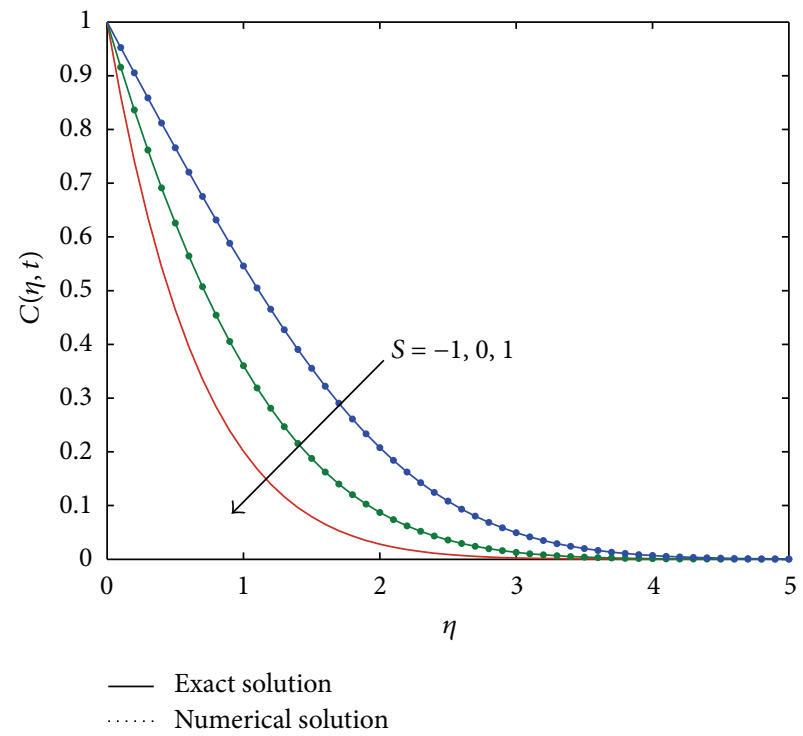

(b)

FIGURE 1: Comparison of numerical and exact solutions of (a) $T(\eta, t)$ and (b) $C(\eta, t)$ for different values of $S$ when $\operatorname{Pr}=0.71, \mathrm{Sc}=1, N=1$, $K_{r}=0.5$, and $t=0.5$.

where $\operatorname{Re}=U_{0} L / v$ is the Reynolds number, $L$ is some characteristic length, and

$$
\begin{aligned}
f(t)=(1+N)[ & \left(\frac{m}{2 \sqrt{n}}+\sqrt{n} t\right) \operatorname{erfc}\left(\sqrt{\frac{n t}{m}}\right) \\
& \left.-\left(\frac{m}{2 \sqrt{n}}+b t+\sqrt{n} t\right)-\sqrt{\frac{m t}{\pi}} e^{-n t / m}\right] .
\end{aligned}
$$

The values of skin friction $\tau$ can be obtained numerically using the Matlab INVLAP routine.

\section{Validation of Numerical Results}

In order to validate the numerical results obtained using the Matlab INVLAP routine, the values of $\mathrm{Nu} / \mathrm{Re}$ are compared with the exact values obtained by Seth et al. [37] in Table 1 . Further, the numerical values of $\mathrm{Nu} / \mathrm{Re}$ and $\mathrm{Sh} / \mathrm{Re}$ are compared with the exact analytical values in Table 2 . The fluid temperature and species concentration profiles are plotted using both the numerical and exact values and are compared 


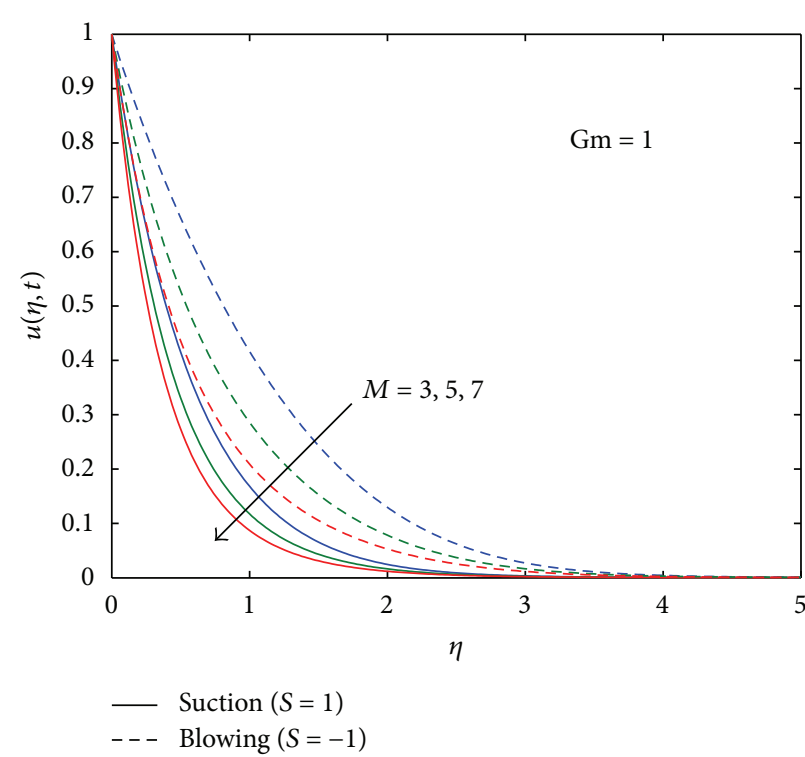

(a)

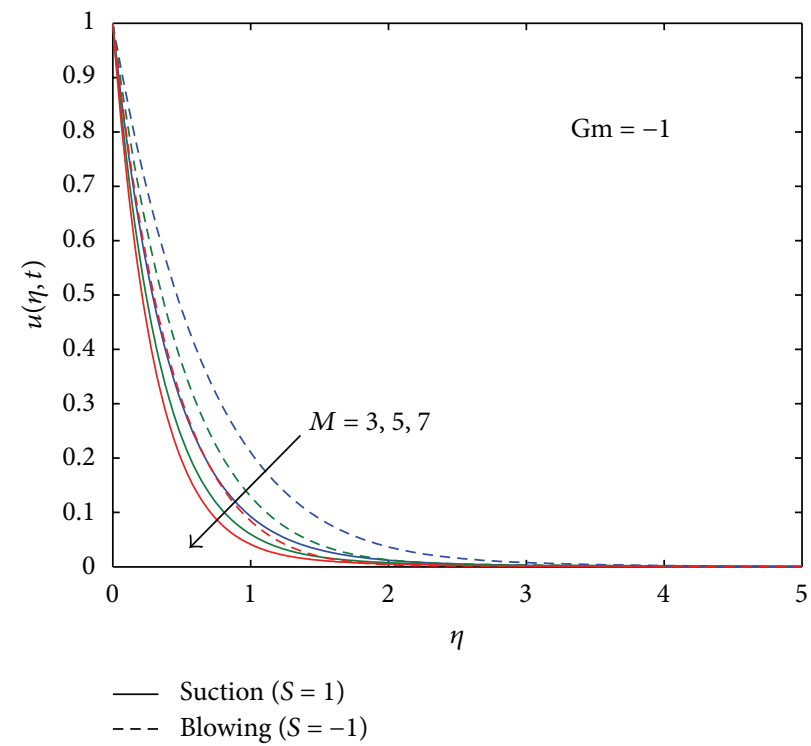

(b)

Figure 2: Effect of $M$ on $u(\eta, t)$ when $\mathrm{Sc}=1, K_{r}=0.5, N=1, \operatorname{Pr}=0.71$, and $t=0.7$.

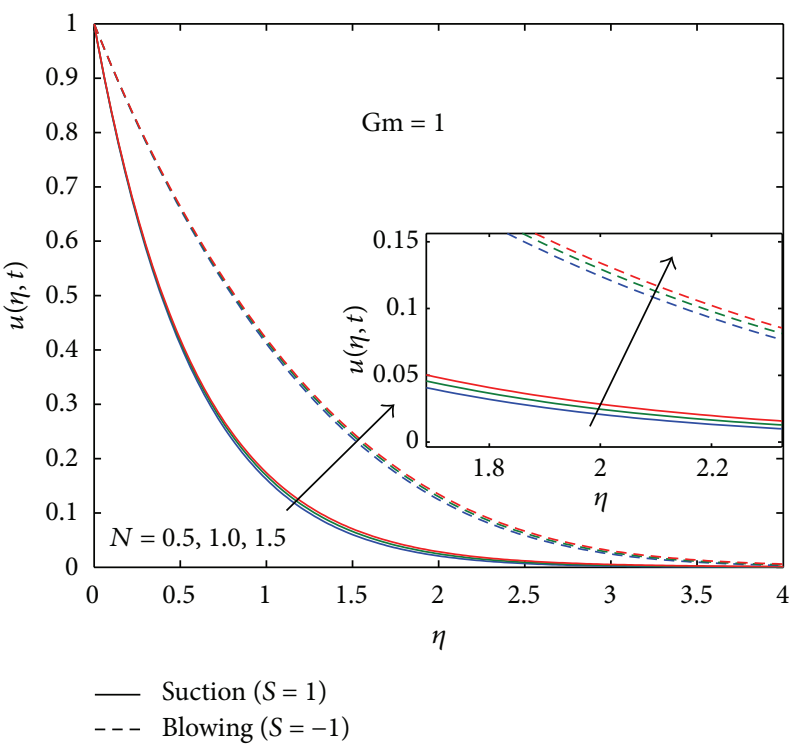

(a)

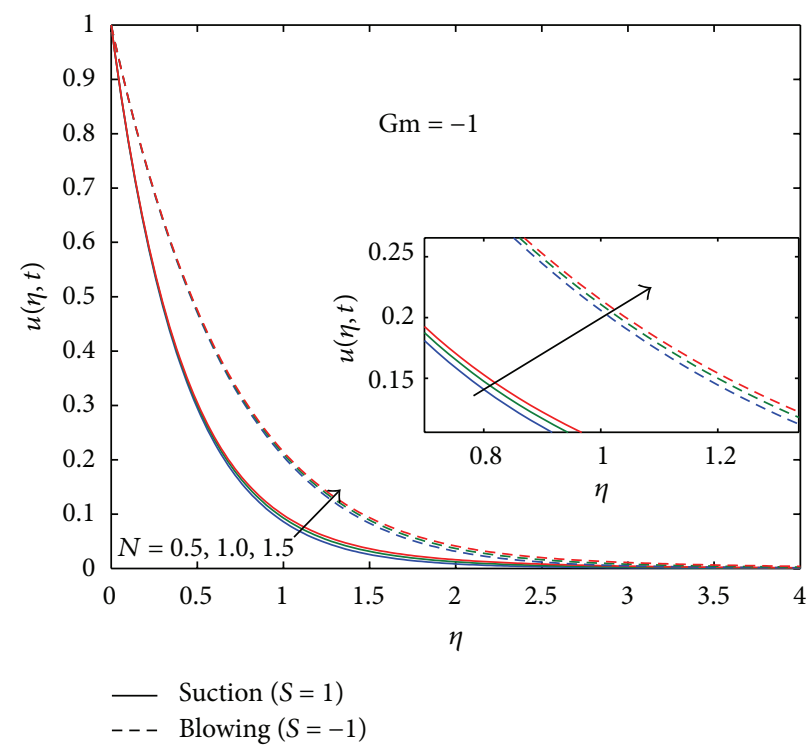

(b)

Figure 3: Effect of $N$ on $u(\eta, t)$ when $\mathrm{Sc}=1, K_{r}=0.5, M=3, \operatorname{Pr}=0.71$, and $t=0.7$.

in Figure 1. An excellent agreement between the values is observed which validates the accuracy of the INVLAP results.

\section{Results and Discussion}

The unsteady hydromagnetic free convective flow of a viscous, incompressible, electrically conducting, and heat radiating fluid past an infinite vertical flat plate in the presence of suction/blowing and chemically reactive species concentration has been studied numerically using the Matlab INVLAP routine. The fluid velocity $u(\eta, t)$, temperature $T(\eta, t)$, and species concentration $C(\eta, t)$ profiles are given in Figures 2, $3,4,5,6,7,8,9,10$, and 11 , whereas the numerical values of the skin friction, heat, and mass transfer coefficients are presented in Tables 3 and 4 when $t=0.7, \mathrm{Gm}=1$ (which corresponds to assisting buoyancy), and $\mathrm{Gm}=-1$ (which corresponds to opposing buoyancy).

Figures 2 to 7 show the effects of the magnetic field, thermal radiation, suction/blowing, chemical reaction, mass diffusion, and thermal diffusion on the fluid velocity. With assisting mass buoyancy (i.e., $\mathrm{Gm}=1$ ), the fluid velocity 


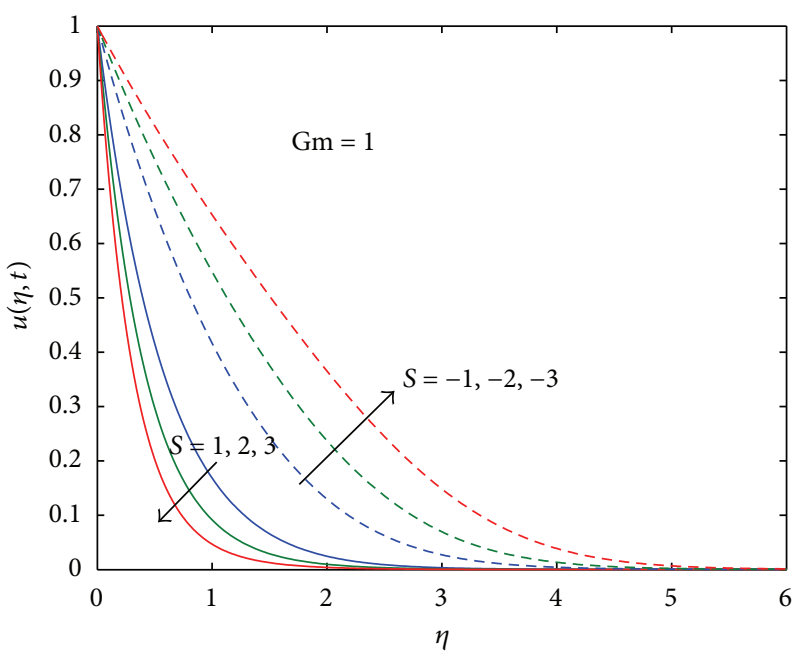

(a)

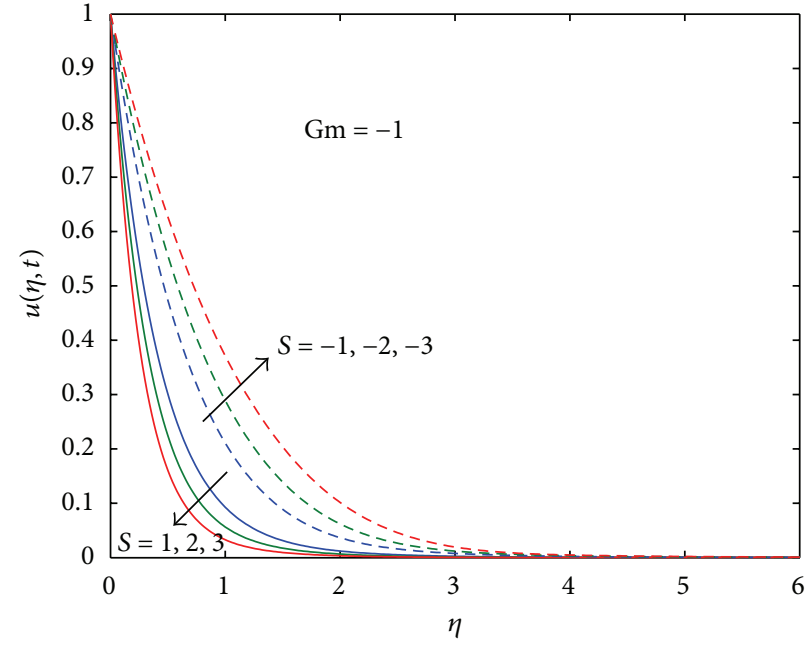

(b)

Figure 4: Effect of $S$ on $u(\eta, t)$ when $\mathrm{Sc}=1, K_{r}=0.5, N=1, \operatorname{Pr}=0.71, M=3$, and $t=0.7$.

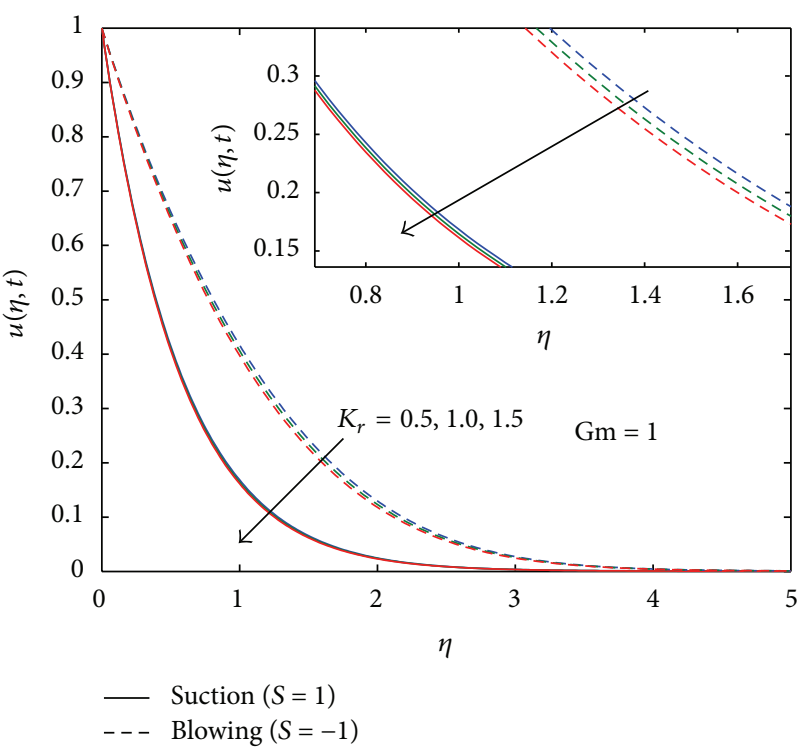

(a)

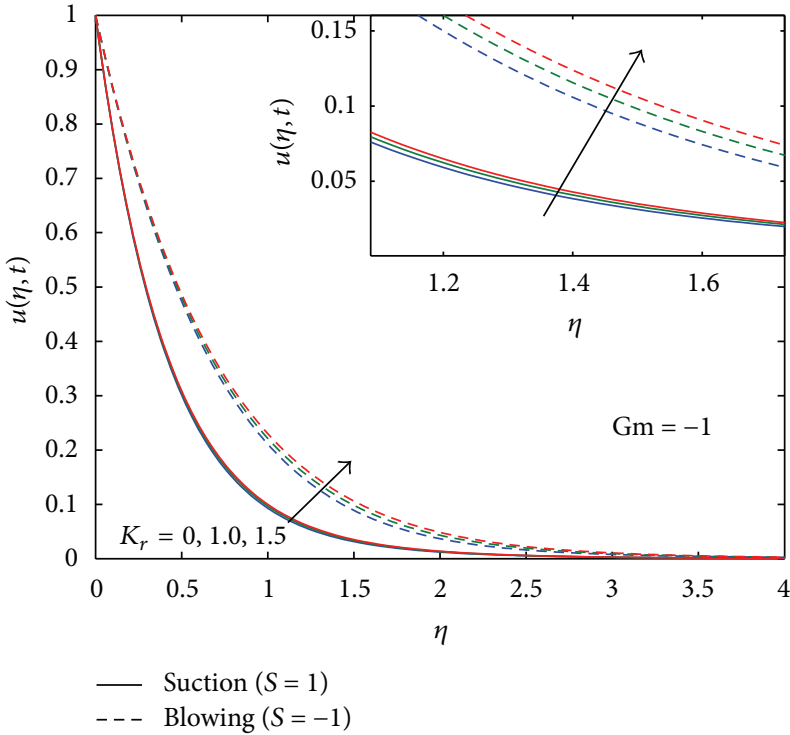

(b)

Figure 5: Effect of $K_{r}$ on $u(\eta, t)$ when $\mathrm{Sc}=1, M=3, N=1, \operatorname{Pr}=0.71$, and $t=0.7$.

decreases with an increase in the magnetic parameter $M$, chemical reaction parameter $K_{r}$, Schmidt number Sc, and Prandtl number Pr while it increases with the thermal radiation parameter $N$. Since the Schmidt number Sc is the ratio of viscous to mass diffusivity, an increase in Sc implies a decrease in the mass diffusion rate. Hence, the magnetic field and the chemical reaction rate tend to reduce the fluid velocity, whereas the mass diffusivity, thermal diffusivity, and thermal radiation tend to increase the fluid velocity. With opposing mass buoyancy (i.e., for $\mathrm{Gm}=-1$ ), the behaviour of the fluid velocity with respect to all governing parameters remains the same except with respect to Sc, when the fluid velocity decreases with an increase in mass diffusivity.
Figure 4 shows that the fluid velocity decreases with an increase in suction velocity, whereas it increases with an increase in blowing for both assisting and opposing mass buoyancy. This result may be useful in engineering applications where the formation of boundary layer is to be delayed. This can be done by increasing the suction velocity. We also observe that the thickness of the momentum boundary layer is less in the presence of opposing mass buoyancy than in the presence of assisting mass buoyancy.

Figures 8,9 , and 10 show the effects of thermal radiation, thermal diffusion, and suction/blowing on the fluid temperature. We observe from Figures 8 and 9 that the fluid temperature increases with $N$ while it decreases with Pr. The 


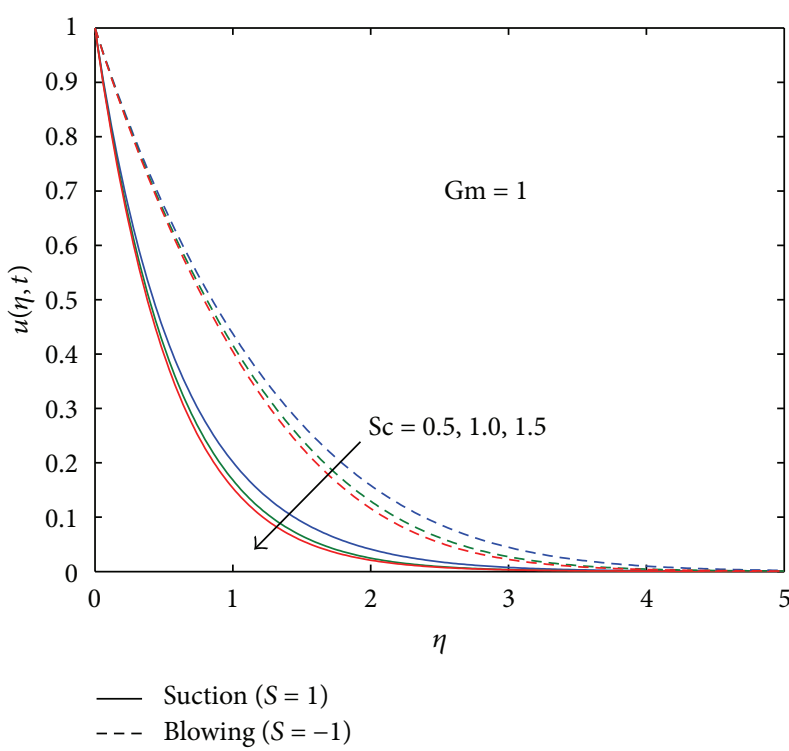

(a)

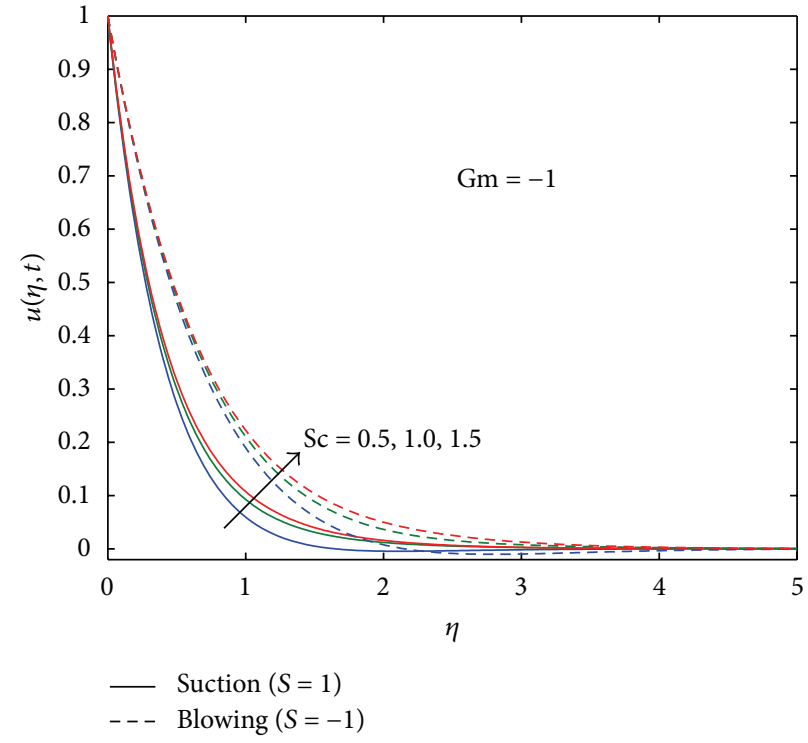

(b)

Figure 6: Effect of Sc on $u(\eta, t)$ when $M=3, K_{r}=0.5, N=1, \operatorname{Pr}=0.71$, and $t=0.7$.

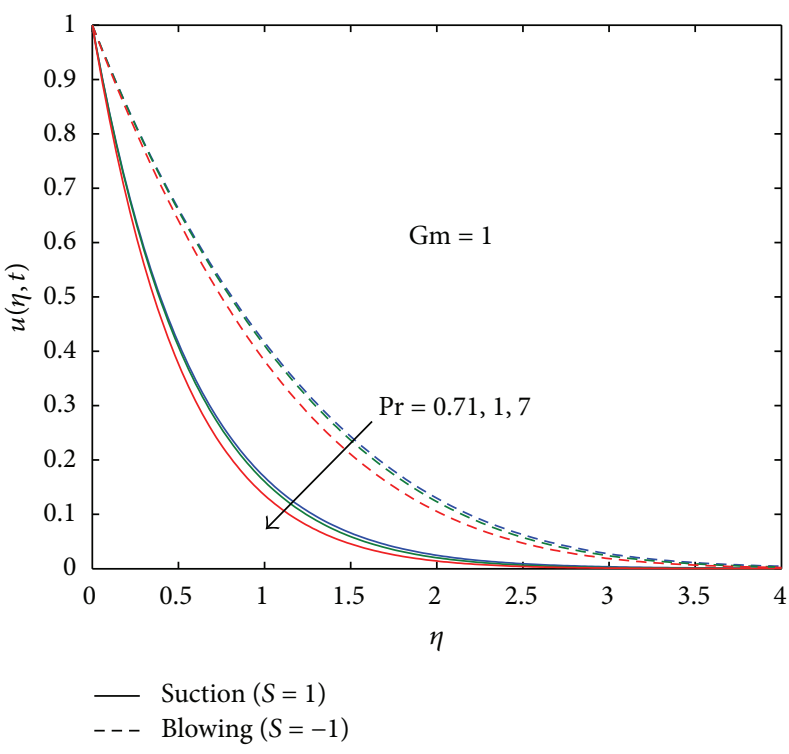

(a)

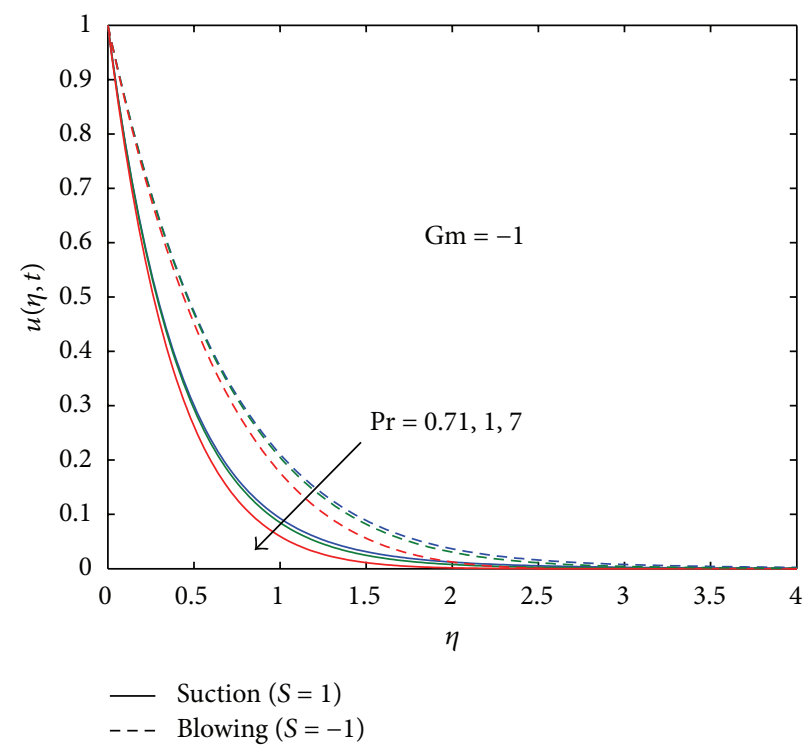

(b)

Figure 7: Effect of $\operatorname{Pr}$ on $u(\eta, t)$ when $\mathrm{Sc}=1, K_{r}=0.5, N=1, M=3$, and $t=0.7$.

Prandtl number is the ratio of viscosity to thermal diffusivity and an increase in Pr implies a decrease in thermal diffusivity. Thermal radiation and diffusivity tend to increase the fluid temperature. Figure 10 shows that an increase in suction velocity causes a reduction in the fluid temperature, whereas an increase in blowing increases the fluid temperature.

Figures 11, 12, and 13 show the effects of the chemical reaction, mass diffusion, and suction or blowing on the species concentration. We observe from Figures 11 and 12 that the species concentration decreases with an increase in the chemical reaction parameter or the Schmidt number. Thus, the chemical reaction rate tends to reduce the species concentration, whereas mass diffusivity increases the species concentration. It is clear from Figure 13 that the suction velocity reduces the species concentration while blowing causes an increase in the species concentration. We observe from Figures 11 and 12 that the species concentration is higher in the case of blowing than in the case of suction. We also observe from Figures 2-11 that the momentum, thermal and concentration boundary layers are thicker in the case of blowing than in the case of suction.

Table 3 shows the effects of the magnetic field, thermal radiation, suction/blowing, chemical reaction, mass diffusion and thermal diffusion on the skin friction in the presence 
TABLE 3: Effect of fluid and physical parameters on the skin friction $-\tau$ when $t=0.7$.

\begin{tabular}{|c|c|c|c|c|c|c|c|c|c|}
\hline$M$ & $N$ & $S$ & $K_{r}$ & Sc & $\operatorname{Pr}$ & $\begin{array}{c}-\tau(S>0 \\
\mathrm{Gm}=1)\end{array}$ & $\begin{array}{c}-\tau(S<0 \\
\mathrm{Gm}=1)\end{array}$ & $\begin{array}{l}-\tau(S>0, \\
\mathrm{Gm}=-1)\end{array}$ & $\begin{array}{l}-\tau(S<0, \\
\mathrm{Gm}=-1)\end{array}$ \\
\hline 3 & 1 & - & 0.5 & 1 & 0.71 & 1.747428 & 0.788854 & 2.397344 & 1.438770 \\
\hline 5 & - & - & - & - & - & 2.291296 & 1.325411 & 2.865812 & 1.899927 \\
\hline 7 & - & - & - & - & - & 2.737296 & 1.766244 & 3.258082 & 2.287031 \\
\hline- & 0.5 & - & - & - & - & 1.763388 & 0.794076 & 2.413303 & 1.443992 \\
\hline- & 1.0 & - & - & - & - & 1.747428 & 0.788854 & 2.397344 & 1.438770 \\
\hline- & 1.5 & - & - & - & - & 1.735676 & 0.784937 & 2.385592 & 1.434853 \\
\hline- & - & 1 & - & - & - & 1.747428 & - & 2.397344 & - \\
\hline- & - & 2 & - & - & - & 2.463830 & - & 3.043645 & - \\
\hline- & - & 3 & - & - & - & 3.291595 & - & 3.786658 & - \\
\hline- & - & -1 & - & - & - & - & 0.788854 & - & 1.438770 \\
\hline- & - & -2 & - & - & - & - & 0.540588 & - & 1.120403 \\
\hline- & - & -3 & - & - & - & - & 0.393845 & - & 0.888908 \\
\hline- & - & - & 0.5 & - & - & 1.747428 & 0.788854 & 2.397344 & 1.438770 \\
\hline- & - & - & 1.0 & - & - & 1.761672 & 0.803098 & 2.383100 & 1.424526 \\
\hline- & - & - & 1.5 & - & - & 1.774216 & 0.815642 & 2.370555 & 1.411981 \\
\hline- & - & - & - & 0.5 & - & 1.666559 & 0.771682 & 2.478212 & 1.455942 \\
\hline- & - & - & - & 1.0 & - & 1.747428 & 0.788854 & 2.397344 & 1.438770 \\
\hline- & - & - & - & 1.5 & - & 1.796602 & 0.798486 & 2.348170 & 1.429138 \\
\hline- & - & - & - & - & 0.71 & 1.747428 & 0.788854 & 2.397344 & 1.438770 \\
\hline - & - & - & - & - & 1.0 & 1.766522 & 0.795089 & 2.416438 & 1.445005 \\
\hline- & - & - & - & - & 7.0 & 1.884422 & 0.830142 & 2.534338 & 1.480058 \\
\hline
\end{tabular}

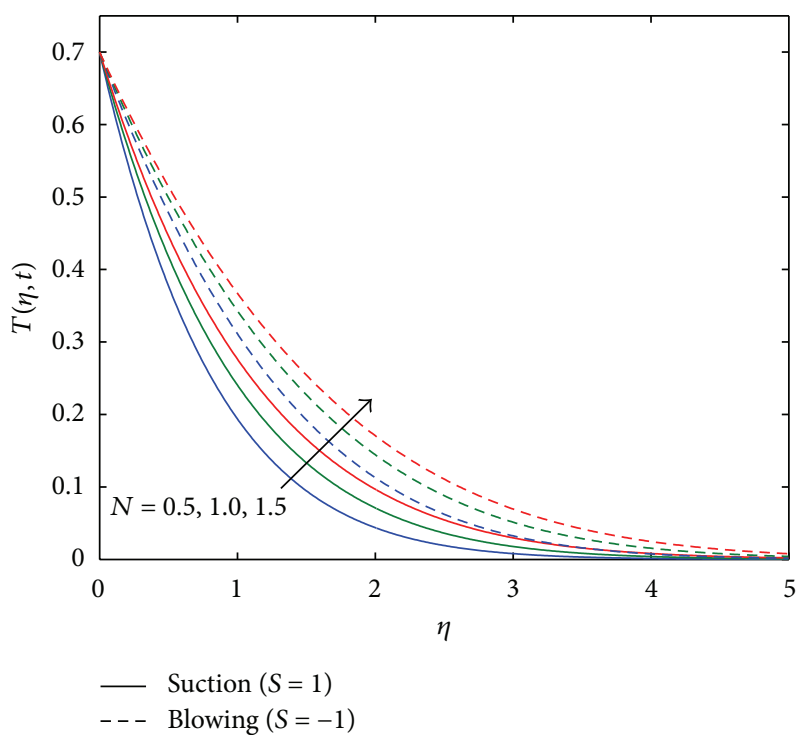

Figure 8: Effect of $N$ on $T(\eta, t)$ when $\operatorname{Pr}=0.71$ and $t=0.7$.

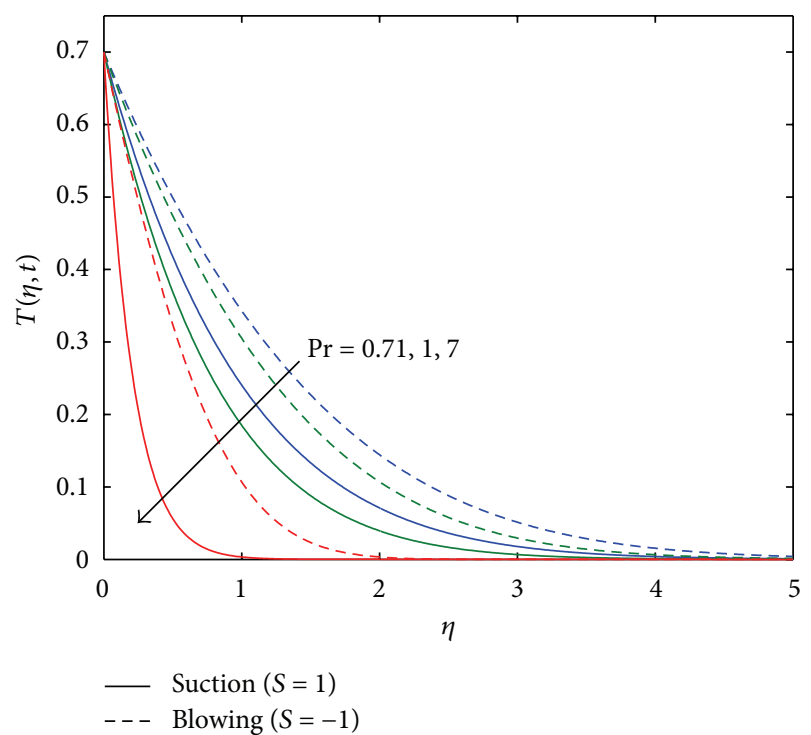

Figure 9: Effect of $\operatorname{Pr}$ on $T(\eta, t)$ when $N=1$ and $t=0.7$. of assisting and opposing mass buoyancy. In the case of assisting mass buoyancy, an increase in $M, K_{r}$, Sc and $\mathrm{Pr}$ tends to increase the skin friction whereas an increase in $N$ reduces the skin friction for both suction and blowing cases. Thus the skin friction increases with an increase in the magnetic field parameter and the chemical reaction rate while it decreases with an increase in mass diffusivity, thermal diffusivity, and thermal radiation in the case of assisting mass buoyancy. However, for opposing mass buoyancy, the skin friction increases with mass diffusivity while decreasing with an increase in the rate of the chemical reaction. 
TABLE 4: Values of $\mathrm{Nu} / \mathrm{Re}$ and $\mathrm{Sh} / \mathrm{Re}$ for different values of governing parameters when $t=0.7$.

\begin{tabular}{|c|c|c|c|c|c|c|c|c|}
\hline$N$ & $S$ & $\operatorname{Pr}$ & $K_{r}$ & Sc & $\begin{array}{c}\mathrm{Nu} / \mathrm{Re} \\
\text { (Suction) }\end{array}$ & $\begin{array}{c}\mathrm{Nu} / \mathrm{Re} \\
\text { (Blowing) }\end{array}$ & $\begin{array}{c}\mathrm{Sh} / \mathrm{Re} \\
\text { (Suction) }\end{array}$ & $\begin{array}{c}\text { Sh/Re } \\
\text { (Blowing) }\end{array}$ \\
\hline 0.5 & - & 0.71 & - & - & 1.249449 & 0.752449 & - & - \\
\hline 1.0 & - & 0.71 & - & - & 1.396642 & 0.899642 & - & - \\
\hline 1.5 & - & 0.71 & - & - & 1.527010 & 1.030010 & - & - \\
\hline 1 & 1 & 0.71 & 0.5 & 1.0 & 1.396642 & - & 1.500355 & - \\
\hline 1 & 2 & 0.71 & 0.5 & 1.0 & 1.712939 & - & 2.280316 & - \\
\hline 1 & 3 & 0.71 & 0.5 & 1.0 & 2.069304 & - & 3.174187 & - \\
\hline 1 & -1 & 0.71 & 0.5 & 1.0 & - & 0.899642 & - & 0.500355 \\
\hline 1 & -2 & 0.71 & 0.5 & 1.0 & - & 0.718939 & - & 0.280316 \\
\hline 1 & -3 & 0.71 & 0.5 & 1.0 & - & 0.578304 & - & 0.174187 \\
\hline 1 & - & 0.71 & - & - & 1.396642 & 0.899642 & - & - \\
\hline 1 & - & 1.0 & - & - & 1.723721 & 1.023721 & - & - \\
\hline 1 & - & 7.0 & - & - & 6.662968 & 1.762968 & - & - \\
\hline- & - & - & 0.5 & 1 & - & - & 1.500355 & 0.500355 \\
\hline- & - & - & 1.0 & 1 & - & - & 1.691322 & 0.691322 \\
\hline- & - & - & 1.5 & 1 & - & - & 1.865496 & 0.865496 \\
\hline- & - & - & 0.5 & 0.5 & - & - & 0.921462 & 0.421462 \\
\hline- & - & - & 0.5 & 1.0 & - & - & 1.500355 & 0.500355 \\
\hline- & - & - & 0.5 & 1.5 & - & - & 2.035801 & 0.535801 \\
\hline
\end{tabular}

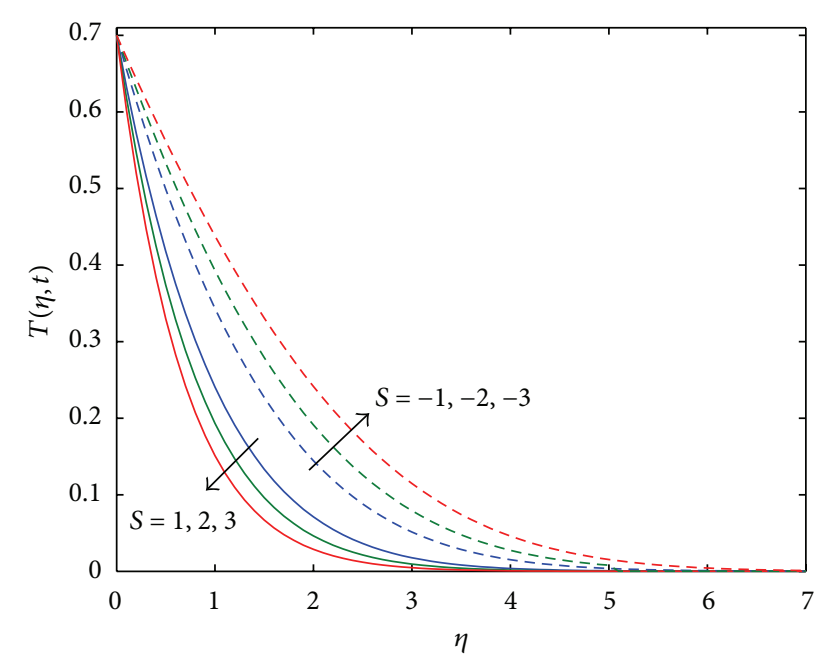

Figure 10: Effect of $S$ on $T(\eta, t)$ when $\operatorname{Pr}=0.71, N=1$, and $t=0.7$.

Table 4 shows the effects of thermal radiation, suction/blowing, and thermal diffusion on the heat transfer rate from the plate. It also shows the effects of suction/blowing, chemical reaction rate, and mass diffusion on the rate of mass transfer at the plate. We observe that the Nusselt number increases with $N$ and Pr. Thus, the thermal radiation tends to accelerate heat transfer at the plate while thermal diffusion has reverse effect. Table 4 also shows that the rate of heat transfer at the plate increases with an increase in suction velocity and decreases with blowing. The Sherwood number increases with $K_{r}$ and Sc for both suction and blowing. An increase in the suction velocity causes an increase in the rate of mass transfer, whereas an increase in blowing has the opposite effect.

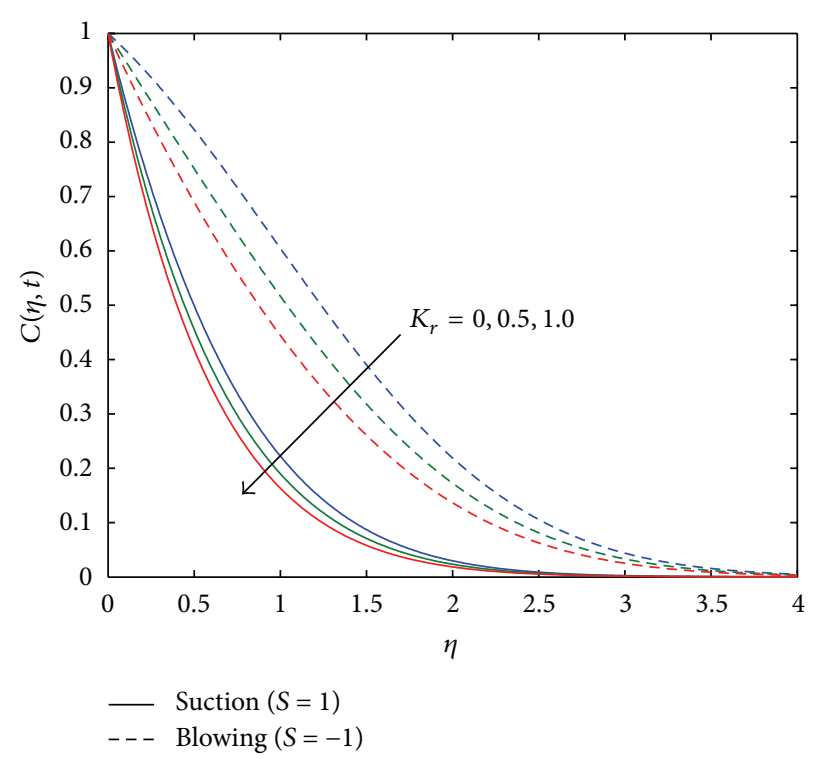

Figure 11: Effect of $M$ on $C(\eta, t)$ when Sc $=1$ and $t=0.7$.

\section{Conclusions}

The unsteady hydromagnetic free convection flow of a viscous, incompressible, and heat-radiating fluid past an impulsively started vertical porous flat plate with ramped temperature in the presence of a chemically reactive species concentration was studied. For certain parameter values, exact solutions of the model equations were found using Laplace transforms. The Matlab INVLAP routine was used to find the numerical results in the general case. The numerical results were found to be in excellent agreement with the 


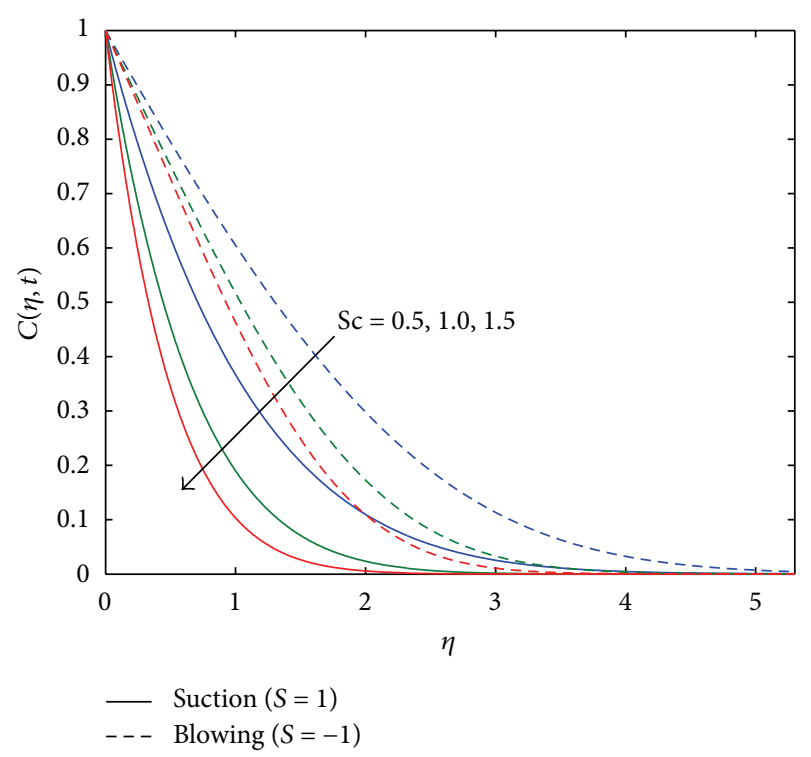

FIgURE 12: Effect of Sc on $C(\eta, t)$ when $K_{r}=0.5$ and $t=0.7$.

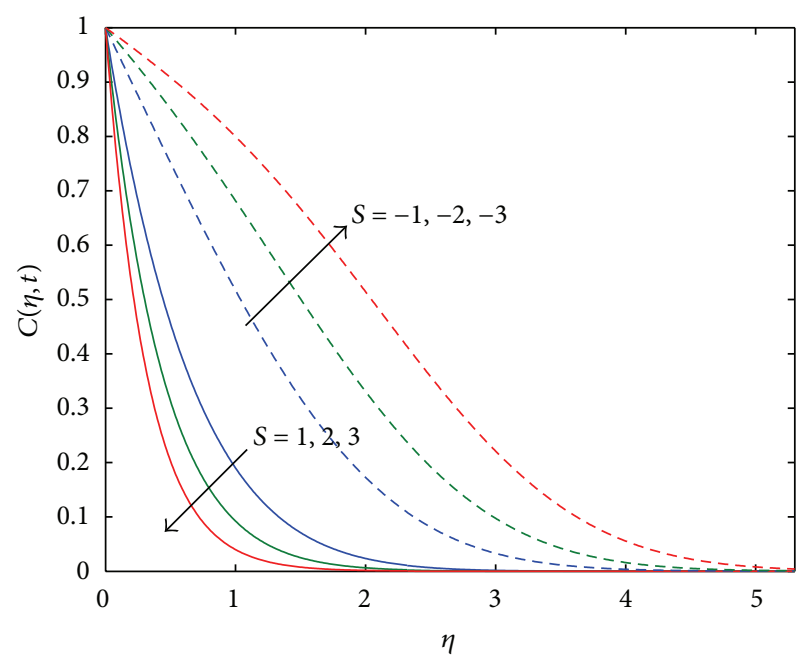

FIGURE 13: Effect of $S$ on $C(\eta, t)$ when $K_{r}=0.5, \mathrm{Sc}=1$, and $t=0.7$.

results of Seth et al. [37] and with the exact results. The important findings of the present work may be summarized as follows.

(i) In the case of assisting mass buoyancy force, the fluid velocity is reduced by an increase in the magnetic field and the chemical reaction rate.

(ii) Blowing causes an acceleration in the fluid velocity while suction has reverse effect on it.

(iii) An increase in the chemical reaction rate tends to reduce the species concentration, whereas the mass diffusivity parameter has reverse effect.

(iv) Thermal radiation, thermal diffusivity, and blowing tend to increase the fluid temperature whereas suction has the opposite effect. (v) In the case of an assisting mass buoyancy force, the magnetic field and chemical reaction parameter tend to increase the skin friction while the mass diffusivity, thermal diffusivity, and thermal radiation have the reverse effect.

(vi) Thermal diffusivity and blowing tend to reduce the rate of heat transfer at the plate whereas thermal radiation and suction have the opposite effect.

\section{Acknowledgment}

The authors would like to thank the referee for his valuable and constructive suggestions.

\section{References}

[1] A. S. Gupta, "Steady and transient free convection of an electrically conducting fluid from a vertical plate in the presence of a magnetic field," Applied Scientific Research, vol. 9, no. 1, pp. 319-333, 1960.

[2] A. S. Gupta, "Laminar free convection flow of an electrically conducting fluid from a vertical plate with uniform surface heat flux and variable wall temperature in the presence of a magnetic field," Zeitschrift für Angewandte Mathematik und Physik, vol.13, pp. 324-333, 1962.

[3] K. R. Cramer, "Several magnetohydrodynamic free convection solutions," Journal of Heat Transfer, vol. 85, pp. 35-40, 1963.

[4] I. Pop, "On the unsteady hydromagnetic free convection flow past a vertical infinite flat plate," Indian Journal of Physics, vol. 43, pp. 196-200, 1969.

[5] H. K. Kuiken, "Magnetohydrodynamic free convection in a strong cross field," Journal of Fluid Mechanics, vol. 40, no. 1, pp. 21-38, 1970.

[6] G. Wilks, "Magnetohydrodynamic free convection about a semi-infinite vertical plate in a strong cross field," Zeitschrift für Angewandte Mathematik und Physik ZAMP, vol. 27, no. 5, pp. 621-631, 1976.

[7] M. A. Hossain, "Effect of hall current on unsteady hydromagnetic free convection flow near an infinite vertical porous plate," Journal of the Physical Society of Japan, vol. 55, no. 7, pp. 21832190, 1986.

[8] T. K. Aldoss, M. A. Al-Nimr, M. A. Jarrah, and B. J. Al-Sha'er, "Magnetohydrodynamic mixed convection from a vertical plate embedded in a porous medium," Numerical Heat Transfer Part A, vol. 28, no. 5, pp. 635-645, 1995.

[9] K. A. Helmy, "MHD unsteady free convection flow past a vertical porous plate," Zeitschrift für Angewandte Mathematik und Mechanik, vol. 78, no. 4, pp. 255-270, 1998.

[10] Y. J. Kim, "Unsteady MHD convective heat transfer past a semiinfinite vertical porous moving plate with variable suction," International Journal of Engineering Science, vol. 38, no. 8, pp. 833-845, 2000.

[11] H. S. Takhar, S. Roy, and G. Nath, "Unsteady free convection flow over an infinite vertical porous plate due to the combined effects of thermal and mass diffusion, magnetic field and Hall currents," Heat and Mass Transfer, vol. 39, no. 10, pp. 825-834, 2003.

[12] N. Ahmed, H. K. Sarmah, and D. Kalita, "Thermal diffusion effect on a three-dimensional MHD free convection with mass transfer flow from a porous vertical plate," Latin American Applied Research, vol. 41, pp. 165-176, 2011. 
[13] M. A. Hossain and H. S. Takhar, "Radiation effect on mixed convection along a vertical plate with uniform surface temperature," Heat and Mass Transfer, vol. 31, no. 4, pp. 243-248, 1996.

[14] A. Y. Bakier and R. S. R. Gorla, "Thermal radiation effect on mixed convection from horizontal surfaces in saturated porous media," Transport in Porous Media, vol. 23, no. 3, pp. 357-363, 1996.

[15] H. S. Takhar, R. S. R. Gorla, and V. M. Soundalgekar, "Radiation effects on MHD free convection flow of a gas past a semi-infinite vertical plate," International Journal of Numerical Methods for Heat and Fluid Flow, vol. 6, no. 2, pp. 77-83, 1996.

[16] A. J. Chamkha, "Thermal radiation and buoyancy effects on hydromagnetic flow over an accelerating permeable surface with heat source or sink," International Journal of Engineering Science, vol. 38, no. 15, pp. 1699-1712, 2000.

[17] G. E.-D. A. Azzam, "Radiation effects on the MHD mixed freeforced convective flow past a semi-infinite moving vertical plate for high temperature differences," Physica Scripta, vol. 66, no. 1, pp. 71-76, 2002.

[18] C. Israel-Cookey, A. Ogulu, and V. B. Omubo-Pepple, "Influence of viscous dissipation and radiation on unsteady MHD free-convection flow past an infinite heated vertical plate in a porous medium with time-dependent suction," International Journal of Heat and Mass Transfer, vol. 46, no. 13, pp. 2305-2311, 2003.

[19] M. A. A. Mahmoud, "Thermal radiation effect on unsteady MHD free convection flow past a vertical plate with temperature-dependent viscosity," Canadian Journal of Chemical Engineering, vol. 87, no. 1, pp. 47-52, 2009.

[20] A. R. Bestman and S. K. Adjepong, "Unsteady hydromagnetic free-convection flow with radiative heat transfer in a rotating fluid-I. Incompressible optically thin fluid," Astrophysics and Space Science, vol. 143, no. 1, pp. 73-80, 1988.

[21] A. J. Chamkha, H. S. Takhar, and V. M. Soundalgekar, "Radiation effects on free convection flow past a semi-infinite vertical plate with mass transfer," Chemical Engineering Journal, vol. 84, no. 3, pp. 335-342, 2001.

[22] M. A. El-Hakiem and M. F. El-Amin, "Thermal radiation effect on non-Darcy natural convection with lateral mass transfer," Heat and Mass Transfer, vol. 37, no. 2-3, pp. 161-165, 2001.

[23] V. R. Prasad, N. B. Reddy, and R. Muthucumaraswamy, "Radiation and mass transfer effects on two-dimensional flow past an impulsively started infinite vertical plate," International Journal of Thermal Sciences, vol. 46, no. 12, pp. 1251-1258, 2007.

[24] O. D. Makinde and A. Ogulu, "The effect of thermal radiation on the heat and mass transfer flow of a variable viscosity fluid past a vertical porous plate permeated by a transverse magnetic field," Chemical Engineering Communications, vol. 195, no. 12, pp. 1575-1584, 2008.

[25] S. Mukhopadhyay and G. C. Layek, "Effects of thermal radiation and variable fluid viscosity on free convective flow and heat transfer past a porous stretching surface," International Journal of Heat and Mass Transfer, vol. 51, no. 9-10, pp. 2167-2178, 2008.

[26] D. Pal, "Heat and mass transfer in stagnation-point flow towards a stretching surface in the presence of buoyancy force and thermal radiation," Meccanica, vol. 44, no. 2, pp. 145-158, 2009.

[27] A. J. Chamkha, "MHD flow of a uniformly streched vertical permeable surface in the presence of heat generation/absorption and a chemical reaction," International Communications in Heat and Mass Transfer, vol. 30, no. 3, pp. 413-422, 2003.
[28] A. A. Afify, "MHD free convective flow and mass transfer over a stretching sheet with chemical reaction," Heat and Mass Transfer, vol. 40, no. 6-7, pp. 495-500, 2004.

[29] A. Postelnicu, "Influence of chemical reaction on heat and mass transfer by natural convection from vertical surfaces in porous media considering Soret and Dufour effects," Heat and Mass Transfer, vol. 43, no. 6, pp. 595-602, 2007.

[30] R. Kandasamy and P. G. Palanimani, "Effects of chemical reactions, heat, and mass transfer on nonlinear magnetohydrodynamic boundary layer flow over a wedge with a porous medium in the presence of ohmic heating and viscous dissipation," Journal of Porous Media, vol. 10, no. 5, pp. 489-501, 2007.

[31] D. Pal and H. Mondal, "Effects of Soret Dufour, chemical reaction and thermal radiation on MHD non-Darcy unsteady mixed convective heat and mass transfer over a stretching sheet," Communications in Nonlinear Science and Numerical Simulation, vol. 16, no. 4, pp. 1942-1958, 2011.

[32] A. A. Hayday, D. A. Bowlus, and R. A. McGraw, "Free convection from a vertical plate with step discontinuities in surface temperature," ASME Journal of Heat Transfer, vol. 89, pp. 244250, 1967.

[33] M. Kelleher, "Free convection from a vertical plate with discontinuous wall temperature," Journal of Heat Transfer, Transactions ASME, vol. 93, no. 4, pp. 349-356, 1971.

[34] T.-T. Kao, "Laminar free convective heat transfer response along a vertical flat plate with step jump in surface temperature," Letters in Heat and Mass Transfer, vol. 2, no. 5, pp. 419-428, 1975.

[35] S. Lee and M. M. Yovanovich, "Laminar natural convection from a vertical plate with a step change in wall temperature," Journal of Heat Transfer, vol. 113, no. 2, pp. 501-504, 1991.

[36] P. Chandran, N. C. Sacheti, and A. K. Singh, "Natural convection near a vertical plate with ramped wall temperature," Heat and Mass Transfer, vol. 41, no. 5, pp. 459-464, 2005.

[37] G. S. Seth, M. S. Ansari, and R. Nandkeolyar, "MHD natural convection flow with radiative heat transfer past an impulsively moving plate with ramped wall temperature," Heat and Mass Transfer, vol. 47, no. 5, pp. 551-561, 2011.

[38] G. S. Seth, R. Nandkeolyar, and M. S. Ansari, "Effects of thermal radiation and rotation on unsteady hydromagnetic free convection flow past an impulsively moving vertical plate with ramped temperature in a porous medium," Journal of Applied Fluid Mechanics, vol. 6, no. 1, pp. 27-38, 2013.

[39] O. D. Makinde, "Free convection flow with thermal radiation and mass transfer past a moving vertical porous plate," International Communications in Heat and Mass Transfer, vol. 32, no. 10, pp. 1411-1419, 2005.

[40] I. U. Mbeledogu and A. Ogulu, "Heat and mass transfer of an unsteady MHD natural convection flow of a rotating fluid past a vertical porous flat plate in the presence of radiative heat transfer," International Journal of Heat and Mass Transfer, vol. 50, no. 9-10, pp. 1902-1908, 2007.

[41] A. A. Afify, "Similarity solution in MHD: effects of thermal diffusion and diffusion thermo on free convective heat and mass transfer over a stretching surface considering suction or injection," Communications in Nonlinear Science and Numerical Simulation, vol. 14, no. 5, pp. 2202-2214, 2009.

[42] K. Cramer and S. Pai, Magnetofluid Dynamics for Engineers and Applied Physicists, McGraw Hill, New York, NY, USA, 1973.

[43] R. C. Meyer, "On reducing aerodynamic heat-transfer rates by magnetohydrodynamic techniques," Journal of Aero Space Science, vol. 25, pp. 561-572, 1958. 


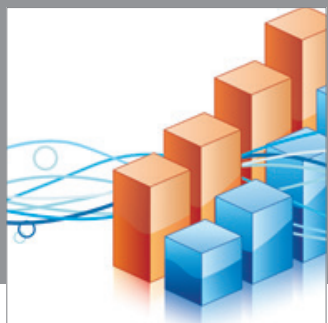

Advances in

Operations Research

mansans

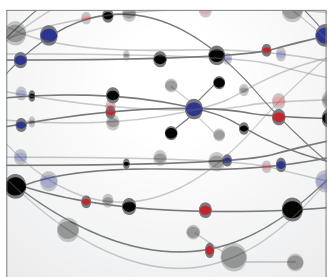

The Scientific World Journal
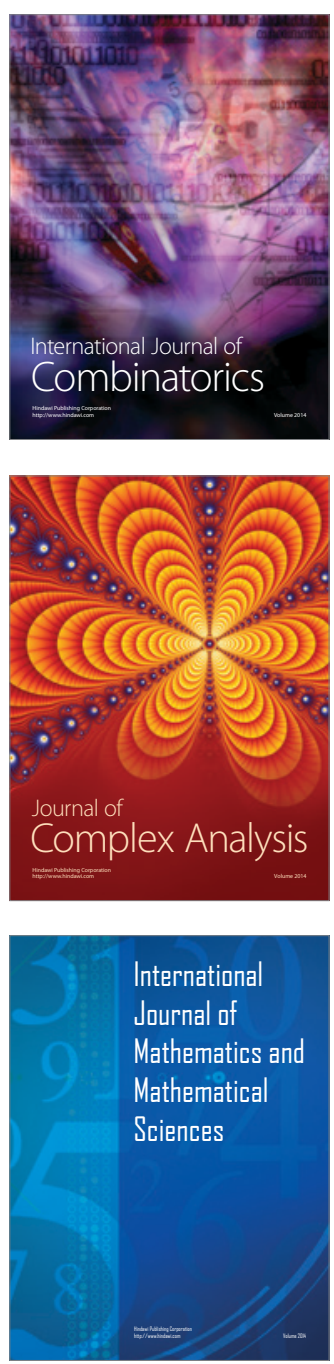
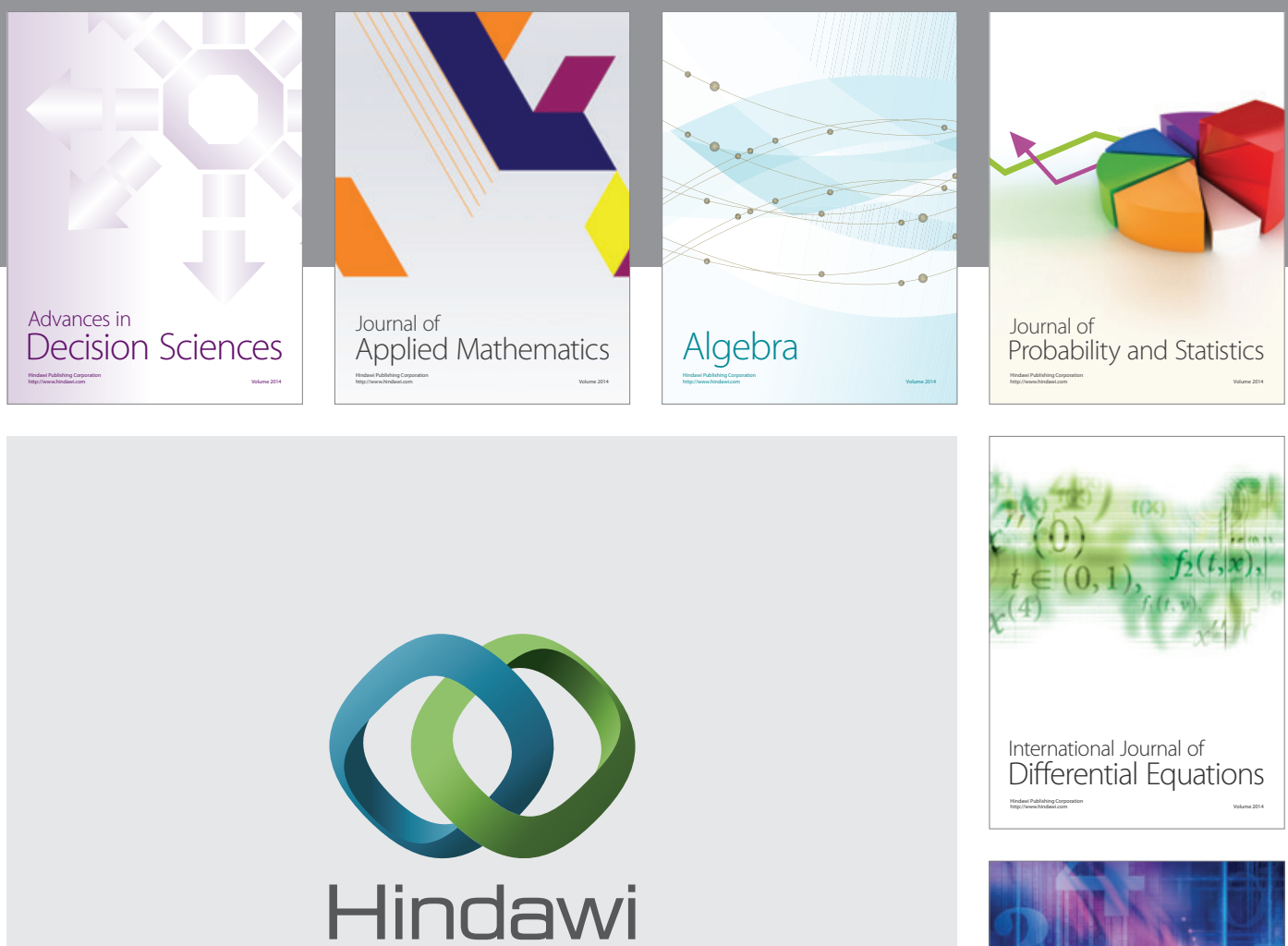

Submit your manuscripts at http://www.hindawi.com
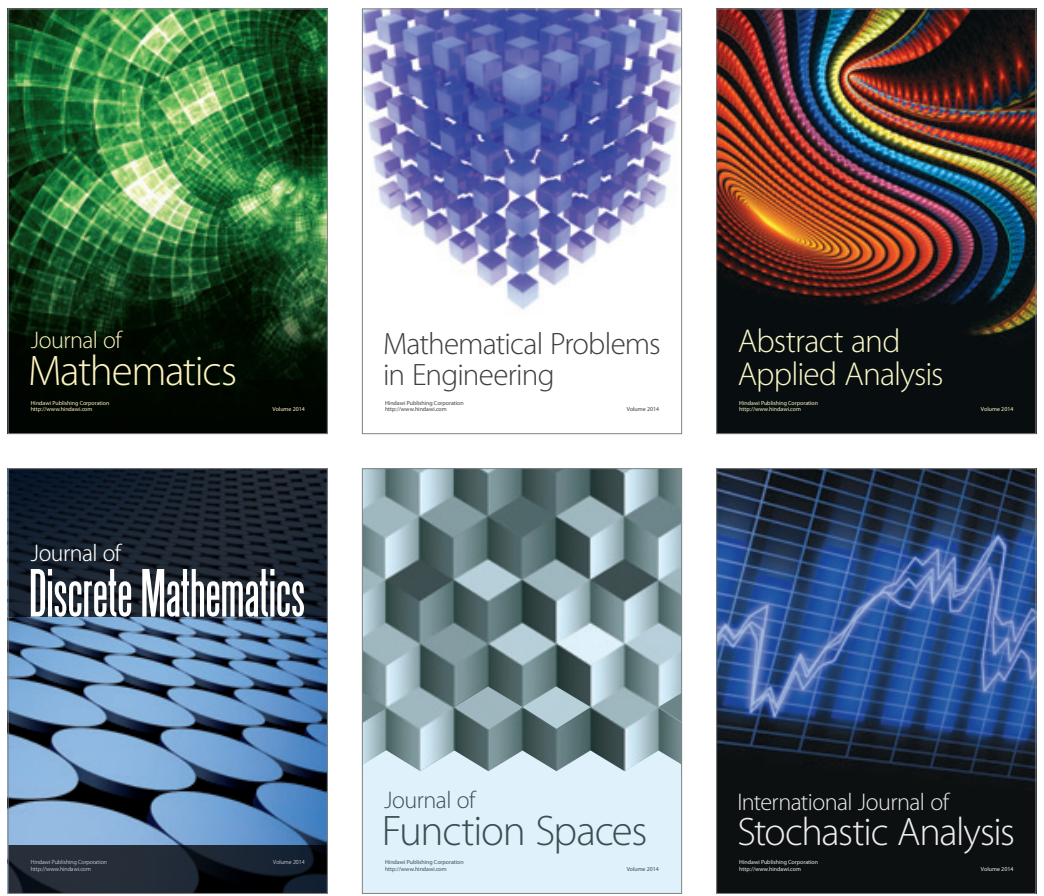

Journal of

Function Spaces

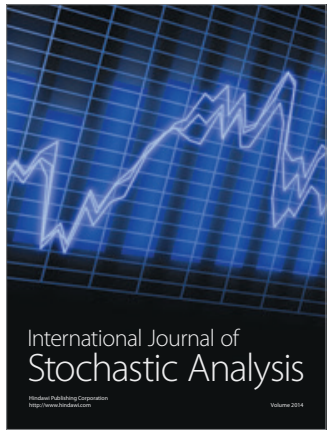

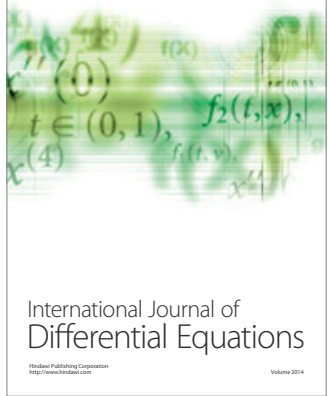
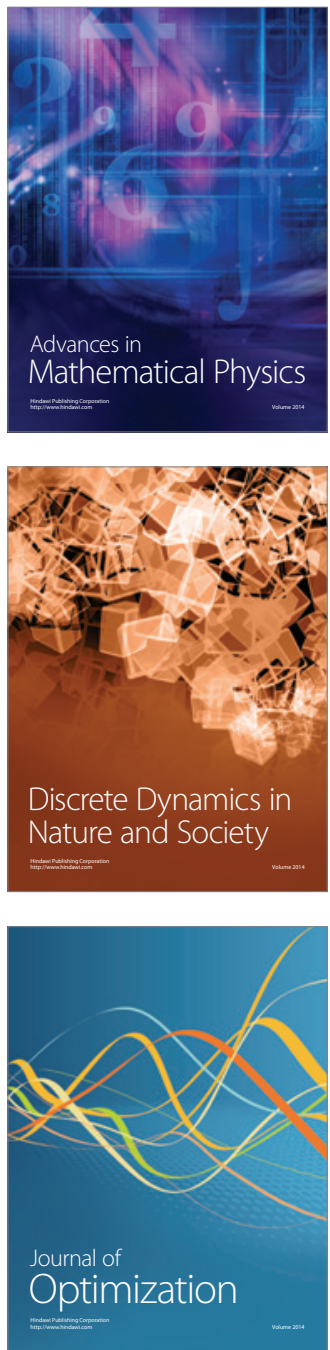